eCommons@AKU

January 2009

\title{
PO5.53 critical illness polyneuropathy and myopathy: experience at a tertiary care center in Pakistan
}

Ghulam Shabbir

Aga Khan University

Ali Mehmood Raufi

Aga Khan University

Mustafa Khan

Aga Khan University

Nawal Salahuddin

Aga Khan University

Saad Shafqat

Aga Khan University, saad.shafqat@aku.edu

Follow this and additional works at: https://ecommons.aku.edu/pakistan_fhs_mc_med_med Part of the Neurology Commons

\section{Recommended Citation}

Shabbir, G., Raufi, A., Khan, M., Salahuddin, N., Shafqat, S. (2009). PO5.53 critical illness polyneuropathy and myopathy: experience at a tertiary care center in Pakistan. Clinical Neurophysiology, 120(1), S59.

Available at: https://ecommons.aku.edu/pakistan_fhs_mc_med_med/545 
keeping with right peripheral facial nerve lesion. Laboratory findings were consistent with polycythemia rubra vera.

Conclusions: Although Bell's palsy in our patient may have occurred by chance, a neuropathic effect of polycythemia rubra vera on the facial nerve cannot be excluded.

P05.51

Marked Improvement of Hepatitis C Associated Chronic Inflammatory Demyelinating Polyneuropathy with Antiviral Therapy: A Case Report

Ghulam Shabbir*, Nadeem Ahmed, Bhojo A. Khealani, Assadullah, Nadir Ali Syed

Section of Neurology \& Dept. of Neurophysiology, Aga Khan University Hospital, Pakistan

E-mail address: ghulam.shabbir@hotmail.com

Background: CIDP is a progressive or relapsing immune mediated disorder often responsive to corticosetereroids, IV immunoglobulin \& plasma exchange. Hepatitis $C$ is common cause of death in patient having chronic hepatic disorder \& affecting approx. 1 out of 10-20 population of Pakistan. Hepatitis C may be associated with peripheral neuropathies mainly axonal, chronic as well as acute cryoglobulinemia, and association with CIDP is less commonly explained. To date the optimal antiviral treatment of Hepatitis $C$ viral infection is the combination of peg interferon-a with ribavarin.

Case report: 44 years old, male with hepatitis $C$ associated CIDP improved with combination of antiviral therapy like interferon-a and Ribavarin. Five weeks after starting therapy he felt $80 \%$ improvement in symptoms (muscle power \& numbness). Viral eradication was confirmed (HCV PCR negative) after treatment patient became symptoms free. Repeat NCS/EMG turned to be normal except absent bilateral H-reflex (possibly secondary to underlying diabetes mellitus.

Conclusions: We described a patient with hepatitis $C$ associated CIDP that markedly improved with the antiviral therapy. The current case report may be an initiative to do more studies in the different canters, particularly in countries where HCV-infection is more common, like in our country Pakistan.

P05.52

Early Denervation in Patients with Axonal Variant of GBS

Ghulam Shabbir*, Shahid Ali Khan, Bhojo A. Khealani, Nadeem Ahmed, Assadullah, Mustafa Khan

Clinical neurophysiology, Section of Neurology, Dept. of Medicine Aga Khan University Hospital Karachi, Pakistan

E-mail address: ghulam.shabbir@hotmail.com

Background: Guillain-Barré Syndrome (GBS) is an important cause of acute neuropathy. Denervating potentials on needle EMG are hallmark of axonal damage. However, this is time depentant phenomenon. The degeneration of axon depends upon length of axon to be degenerated. Shorter distance will require shorter time similarly denervation also appear early in this situation. Studies claim variable time duration for denervation potentials form two weeks to three weeks. This study was to determine early denervation potentials in axonal GBS.

Methods: We retrospectively reviewed the clinical and neurophysiological data of GBS patients over a period of three years GBS patients were classified according to established neurophysiologic criteria.

Results: Forty-two patients were included, 25 were males and 17 females. Thirteen (30\%) had axonal GBS. Out of thirteen ten (10) underwent early electrodiagnostic study within 14 days and three (3) after 14 days. $4 / 10(40 \%)$ had denervation potentials early in the course of illness (less than fourteen).

Conclusions: Denervation potentials can be seen less than two weeks duration in axonal GBS. Further large studies are required to establish whether this has relation to bad outcome in axonal variant of GBS.

\section{P05.53}

Critical Illness Polyneuropathy and Myopathy: Experience at a Tertiary Care Center in Pakistan

Ghulam Shabbir ${ }^{1 *}$, Ali Mehmood Raufi ${ }^{1}$, Mustafa Khan ${ }^{1}$, Nawal Salahuddin ${ }^{2}$, Saad Shafqat ${ }^{1}$

${ }^{1}$ Section of Neurology, Aga Khan University Hospital, Pakistan, ${ }^{2}$ Section of Critical Care Medicine, Aga Khan University Hospital, Pakistan E-mail address: ghulam.shabbir@hotmail.com

Background: Polyneuropathy and Myopathy are frequently encountered in the setting of Critical illness patients. Up to $50 \%$ of intensive care unit (ICU) patients show electrophysiological features of either or both conditions. These conditions interfere with functional recovery and delay weaning from mechanical ventilation, resulting in excess morbidity, mortality and cost of care. This study was to define the clinical spectrum of critical illness Polyneuropathy (CIP) and Myopathy (CIM) in a setting of developing country.

Methods: Hospital records spanning the period 2000 through 2005 were searched with ICD-9 codes to identify patients with CIP and CIM. Functional improvement was judged by (i) increment of at least 1 grade on the Medical Research Council scale of motor strength: and (ii) reappearance of deep-tendon reflexes.

Results: CIP or CIM was established in 47 patients of these, mean age was 54: mean length of stay in hospital 34 (range 8-100) electrophysiological studies revealed CIP in 31 patients (66\%) CIM in $12(26 \%)$ and a mixed picture in $4(8 \%)$. Major co-morbid conditions included sepsis $(39 \%)$ and diabetes mellitus $(17 \%)$, with an additional $28 \%$ having both and $17 \%$ having either. Neuromuscular blocking drugs were administered to 14 (30\%) and steroids to $10(21 \%)$ patients: an additional $11(23 \%)$ patients received both agents while $12(26 \%)$ patients received neither. In hospital mortality was $51 \%$. At discharge and follow-up, motor improvement was seen in $11(23 \%)$ while $12(26 \%)$ remained neurologically unchanged.

Conclusions: We conclude that CIP and CIM are frequent identifiable complications of critical illness in our setting. These observations need further prospective studies to delineate risk factors and out come predictors, to improve critical care patients.

\section{P05.54}

Polycranial Neuropathy and Sensory Ataxia with lgG Anti-GD1a Antibody as a Variant of Guillain-Barré Syndrome

Sun Young $\mathrm{Kim}^{1 *}$, Jong Kuk Kim²

${ }^{1}$ Dept. of Neurology, Kyungpook National University College of Medicine, Korea, ${ }^{2}$ Dept. of Neurology, Kosin University College of Medicine, Korea

E-mail address: advania9@chol.com

Background: IgG anti-GD1a ganglioside antibody is one of the important markers of acute motor axonal neuropathy (AMAN) type Guillain-Barré syndrome (GBS). It is highly associated with disease severity, need for mechanical ventilation and axonal degeneration of peripheral nerve. But, there was limited report presented with cranial neuropathy without significant motor weakness. We report a patient manifested as polycranial neuropathy and sensory ataxia with IgG anti-GD1a antibody without peripheral neuropathy as a variant of GBS.

Case report: A 46-year-old woman visited with slurred speech, swallowing difficulty and gait disturbance following diarrhea which had developed since 2 days ago. Neurological examinations revealed enlarged pupils as $5 / 5 \mathrm{~mm}$ with sluggish light reflexes. Bilateral facial weaknesses, bulbar palsy with decreased gag reflex and neck flexor weakness were noted. The deep tendon reflexes were lost but motor power was normal in all extremities. Decreased sensations of vibration and position in distal limbs were found. She showed ataxic gait with positive Romberg sign. Laboratory studies including routine chemistry, protein electrophoresis, rheumatologic profiles and cerebral spinal fluid examinations were normal. IgM and IgG anti-ganglioside antibodies to GM1, GM2, GD1b, GD3, GT1b, GQ1b and GT1a were all negative except for lgG antiGD1a antibody (1:640). Routine nerve conduction study (NCS) including facial NCS and blink reflex test performed at 3rd, 7th and 13th days of symptoms onset. Blink reflex test at 7th day showed compatible findings with bilateral facial neuropathy, although other studies couldn't reveal any abnormal finding.

Intravenous immunoglobulin treatment was started $(400 \mathrm{mg} / \mathrm{kg} /$ day for 5 days) and her deficits were improved during 2 weeks of admission. At 3rd months for follow up, she showed complete recovery in neurological examinations.

Conclusions: Our case showed multiple cranial neuropathies including facial, bulbar and neck weaknesses in addition to sensory ataxia. These findings suggest that GD1a ganglioside might be located at cranial nerves and sensory nerve root including dorsal root ganglia. This is the first case of GBS with IgG anti-GD1a antibody manifested as polycranial neuropathy and ataxia. 